ich auf drei weitere Gruben (Abb. 6) ron nur 30-40 cm Tiefe, bei einem Durchmesser von $1,30 \mathrm{~m}$, die in fast gleicher Grösse der Herdgrube denselben Inhalt an Knochen und Scherben, einigen Knochengeräten und speckiger, schwarzer Erde hatten. Für Pfostenlöcher halte ich sie zu flach. Es hat fast den Ansclein, als lägen sie rings um den Herd herum. Leider war es mir nicht möglich, die Stelle genauer zu untersuchen, da der Besitzer das Feld bestellen wollte. Wegen unseres schweren Bodens ist er in diesem Jahre auf die wenigen Felder mit leichtem Boden, wie dieses hier, ange-

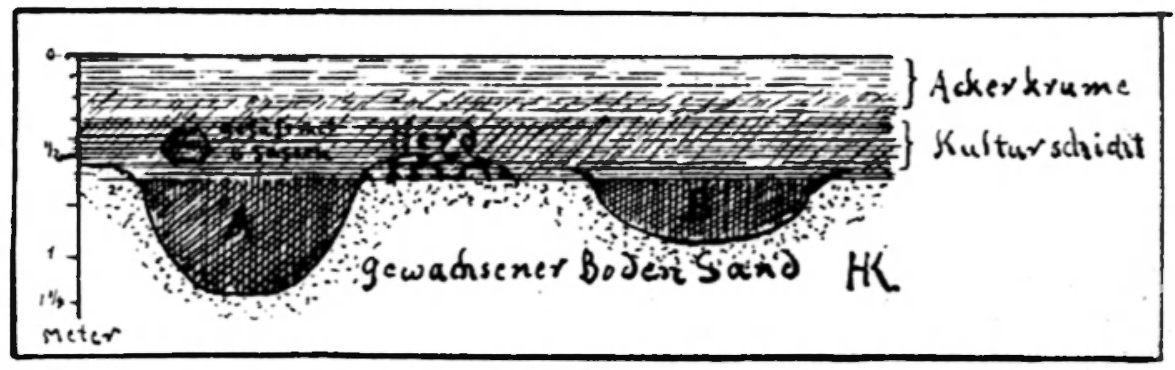

Abl. - . Hohensalza. Querschnitt.

wiesen. Auf der Oberfläche des Feldes farden sich noch zwei Mahlsteine, etwa faustgross, mit abgeschliffener Fläche. Was an Knochen vorkam, weist auf jetzt noch gebräuchliche Haustiere, wie Schwein, Rind, Pferd usw. hin.

Die Grabungen werden an einer anderen Stelle mit Unterstützung des Kreises fortgesetzt, urd es dürfte dabei noch manch wichtiger Fund zutage hommen.

\title{
Ein Dorf aus der Bronzezeit bei Hasenfelde, Kr. Lebus
}

\section{Yon A. Kiekebusch}

Bereits seit dem Frühjahr 1908 ist das Märkische Museum mit Ausgrabungen bei Hasenfelde beschäftigt. Auf dem „Kirchhofsschlag“ des Lehngut sbesitzers Schwarz wurden beim Pfügen Steine aus dem Boden gerissen, und $\mathbf{z w i s c h e n}$ oder unter den Steinen lagen Reste von Gefässen der jüngeren Bronzezeit. Leider waren die Grabanlagen teilweise schon zerstört. Trotzdem erwies sich die Ausbeute noch als ziemlich reich.

Eines der Gräber ist so, wie es gefunden wurde, in der prähistorischen Abteilung des Märkischen Museums ausgestellt (Raum 8, am Eingange von 7 her). Es enthält eine degenerierte Buckelurne, neben der noch ein Scherben lag, der ebenfalls mit Buckeln verziert ist (Abbildung von diesem Grabe im Mannus I. (1909), S. 136, Abb. 4). ${ }^{1}$ )

1) A. Kiekebusch: Die prähistor. Abteilung des Märk. Mus. Mannus I. 1909, S. 130 
Das Gräberfeld bei Hasenfelde (Abb. 1) ist mehrere Morgen gross. Genau haben sich die Grenzen noch nicht feststellen lassen. - Gelegentlich der Ausgrabungen auf dem Urnenfriedhofe. der auf diluvialem Boden liegt, erkundigte ich mich bei Herrn Schwarz, wo die nächste wasse r reiche $\mathrm{N}$ iederung sei. Auf der Karte war eine solche wohl auf der anderen Seite des „Vorderparkes“, neben dem das Gräberfeld sich hinzieht, zu beobachten, schien hier aber zu geringfügig zu sein, um zur Siedlung besonders anzulocken. Auf meine weiteren Fragen erfuhr ich, dass diese mit Gras bewachsene Niederung noch heute den Namen „Seewies e“ führe und dass der Abfluss der Wiese, der wenigstens im Frühjahr noch Wasser führt, ,Seegraben" heisst.

Ich bat Herrn Schwarz und seine Leute, beim Beackern des Feldes in der Umgebung der „Seewiese“ und der „R a d e la n d wi e s e", einer zweiten Niederung, auf etwa hervortretende ,schwarze Stellen“ und vor allem auf zerstreut liegende Scherben zu achten, weil wir auf diese Weise die zum Gräberfelde gehörige Wo hns tät t e finden müssten.

Schon im August 1909 erhielt das Märkische Museum die Nachricht, dass die Wohnstätte gefunden wäre. Ich selber besuchte sogleich den neuen Fundplatz und war überzeugt, dass am Abhange des "Radelandberges" zur „Radelandwiese“ hinunter die Siedlung oder ein Teil von ihr gelegen hatte. Utberall zeigten sich zerstreute Spuren von Kohle, mit Scherben gemischt, auf dem frisch gepflügten Acker. Um die Stellen festzuhalten, nahm ich sie mittels Dreiecksmessung auf und hatte dann nur noch soviel Zeit, zwei der schwarzen Stellen zu untersuchen, wobei sich herausstellte, dass es sich um Herdstellen oder Abfallgruben handelte. Pfostenlöcher waren in der nächsten Umgebung nicht zu finden. Eine Siedlung war hier unbedingt vorhanden; von einem Dorfe wollte ich aber erst reden, wenn die Grundrisse der Häuser festgestellt wären.

$\mathrm{Zu}$ einer gründlichen Untersuchung kam ich, da mich mittlerweile die Arbeiten in Buch in Anspruch nahmen, erst wieder in diesem Herbste. Unterdessen waren auch an anderen Stellen, namentlich an der "Seewiese“ und am „Oberpark" um den Pfuhl herum, der den Namen "Schafiäsche" führt, Siedlungsspuren beobachtet worden.

Durch die anhaltende Dürre war der Boden des Stoppelfeldes an der Radelandwiese so hart geworden, dass der Spaten kaum eindrang. An der "Seewiese" und am „Oberpark" hatte man frisch gepflügt, und so begann ich die Untersuchung an diesen günstigeren Stellen.

Hauptzweck der vom 11.-13. September d. J. vorgenommenen Ausgrabung war es, festzustellen, ob bei Hasenfelde ähnliche $\mathrm{H}$ a usgrund $r$ isse zutage treten würden wie bei Buch. Ich selber war nach allem, was ich vorher beobachtet hatte, davon fest überzeugt und habe ja auch am Schlusse meiner letzten Veröffentlichung über Buch ${ }^{1}$ ) der Ansicht Ausdruck gegeben, dass sehr bald ähnliche Dörfer aufgedeckt werden würden. $\mathrm{Zu}$ jedem grösseren vorgeschichtlichen Gräberfelde

1) P. Z. II, 395. 


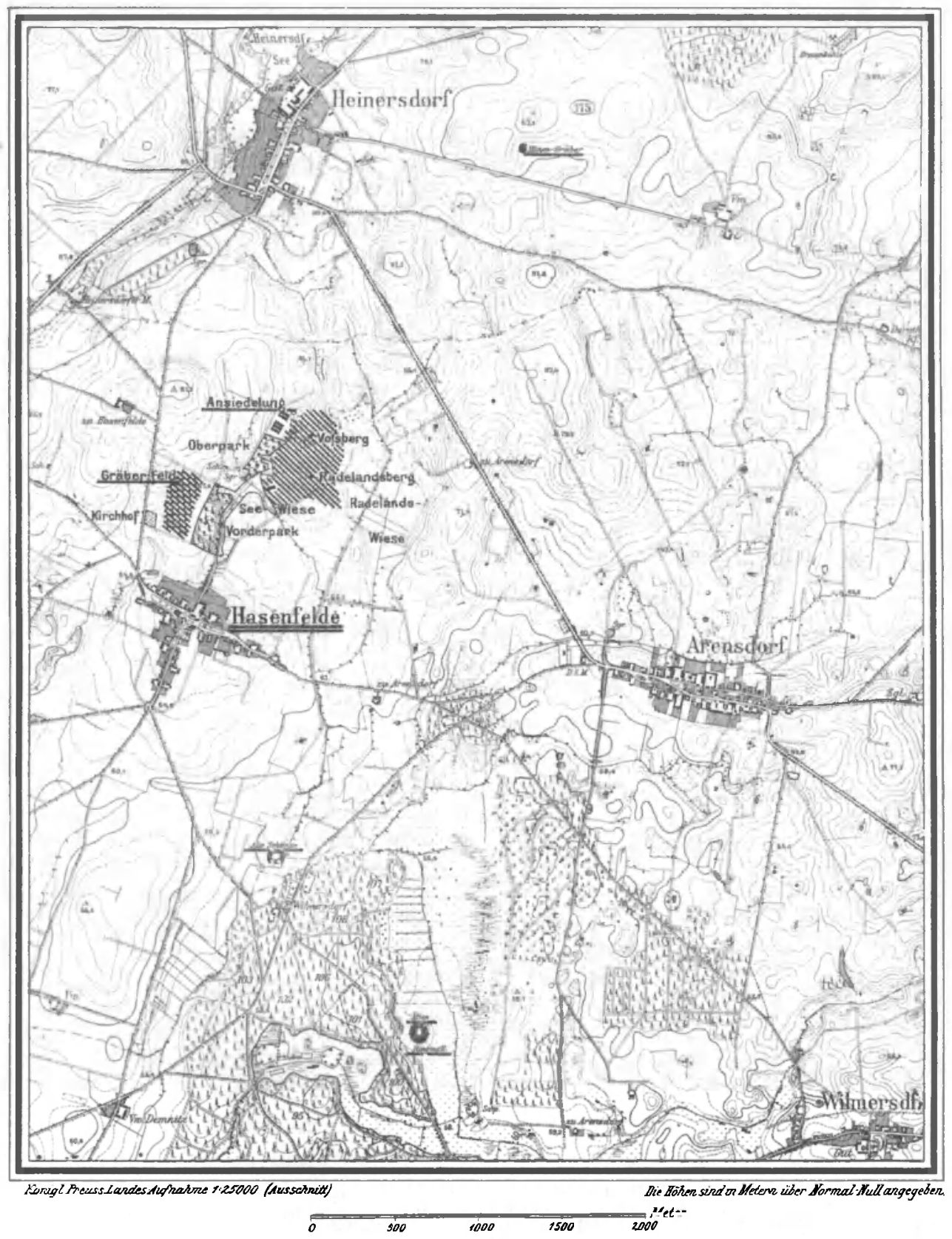

Abb. 1.

Nordöstlich von Hasenfelde liegt ein zweiter Friedhof auf Heinersdorfer Gebiet (,Hünengräber"). Südlich von Hasenfelde zwei Ringwälle, die "Alte Schlange" auf Arensdorfer und der "Alte Ringwall" auf Wilmersdorfer Feldmark. Auf dem "Vossberge" zahlreiche Feuersteinsplitter (Messer und dgl.). Die Grenzen der Ansiedlung und des Gräberfeldes sind noch nicht genau festgestellt und konnten bisher nach den Spuren, die sich fanden, nur vermutungsweise angegeben werden. Die Lage der Grundrisse ist mit I, II u. III bezeichnet. 
muss ein Dorf aus derselben Zeit zu finden sein. Aus Fachkreisen war mir auf diese Behauptung hin mehrfach gesagt worden, ich solle doch nach den Erfolgen bei Buch nun auch Grundrisse mit Pfostenlöchern in der Nähe des von mir untersuchten Friedhofes bei Hasenfelde nachweisen.

Das Ergebnis der Ausgrabung war auch für mich überraschend. In drei Tagen habe ich drei Grundrisse aufgedeckt und untersucht. Man brauchte nur über das Feld zu gehen, so sah man die vom Pfluge aufgeworfene Branderde der $\mathrm{Herdstellen,} \mathrm{ja} \mathrm{selbst} \mathrm{auf} \mathrm{dem} \mathrm{Stoppelfelde} \mathrm{am}$ Abhange des Radelandberges konnte ein geübtes Auge die dunkleren Stellen noch deutlich erkennen. Es ist geradezu erstaunlich, dass auf einem Acker, der sicher seit Jahrhunderten unter dem Pfluge war, sich die doch immerhin nur geringen Reste der Brandspuren noch Jahr für Jahr bemerkbar machen. In Buch ist das erklärlich. Da liegt unter dem Humus die 25-30 cm dicke alte Kulturschicht, von der alljährlich Reste an die Oberfläche befördert werden konnten, ohne dass die Stärke der schwarzen Schicht wesentlich beeinträchtigt wird. Bei Hasenfelde war das Gebiet nie so dicht und dieselbe Stelle nicht mehrfach hintereinander besiedelt, so dass nirgend eine alte Kulturschicht entstand. Das deutliche Hervortreten der schwarzen Spuren erklärt sich hier vielleicht daraus, dass seit einigen Jahren etwas wenn auch nur wenige Zentimeter - tiefer gepflügt wird als früher.

\section{Grundriss I}

Auf dem „Vierweidenschlag“" an der „,Seewiese“ waren mehrere ,schwarze Stellen" zu erkennen. Hier am Abhange konnte sehr wohl ein Haus gestanden haben. Die „Seewiese" war früher sicher ein kleiner „See"; sie bedarf heute noch eines verhälinismässig tiefen Entwässerungsgrabens. Die Siedlung lag auch etwa $15-20 \mathrm{~cm}$ vom Rande der heutigen Wiese entfernt. Zwischen der Wiese und dem Grundriss I habe ich keine weiteren Spuren beobachtet, und es lässt sich vermuten, dass der ehemalige See sich über den Rand der Wiese hinaus ausdehnte.

Der Grundriss I ist beinahe rechteckig (Abb. 2). Durch eine M it t e l w a nd wird die nach der "Seewiese": zu liegende Vorhalle vom Herdraume getrennt. Der H e rd schien zuerst sehr gross zu sein. Steine und Branderde waren augenscheinlich über die ursprüngliche Peripherie hinaus zerstreut und auseinandergetreten worden. Der Kern des Herdes lag mehr nach der Nordostecke zu, also der Nord- und Ostwand näher als der Mittelund Südwand. (Siehe Buch, Grundriss I. P. Z. II, 374.) So blieb auch hier möglichst viel Raum frei für den Aufenthalt der Bewohner und für wirtschaftliche Arbeiten.

Der Herd war aus Steinen gepackt. Der Pflug hatte im Laufe der Zeit sicher schon viele Steine aus ihrer Lage gerissen. Die Packung war nicht mehr unberührt. Trotzdem wurden noch 322 teils faust-, teils kopfgrosse Steine gezählt, die vom Brande völlig geschwärzt, teilweise sogar mürbe waren, so dass man sie auch hier zwischen den Fingern zerreiben konnte. Dass sich auf dem Herde auch Kohlenreste befanden. ist 
selbstverständlich. Ausser zwei grösseren Tierknochen lagen auch Gefässreste, unter ihnen ein Randstück, zwei Eier-oder Schwalbensteine und ein Belemnit.

Die Grube unter dem Herde war vom Planum aus nur noch $20-30 \mathrm{~cm}$ tief. Der kiesige Sand in der Umgebung des Herdes hatte sich durch die
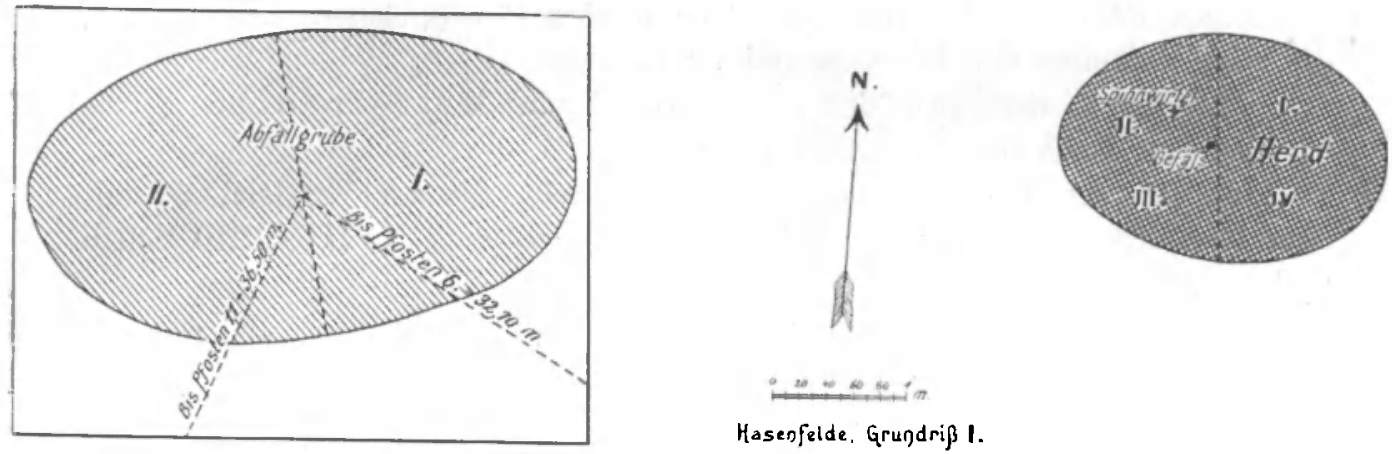

asenfeide. Grundriß 1 .

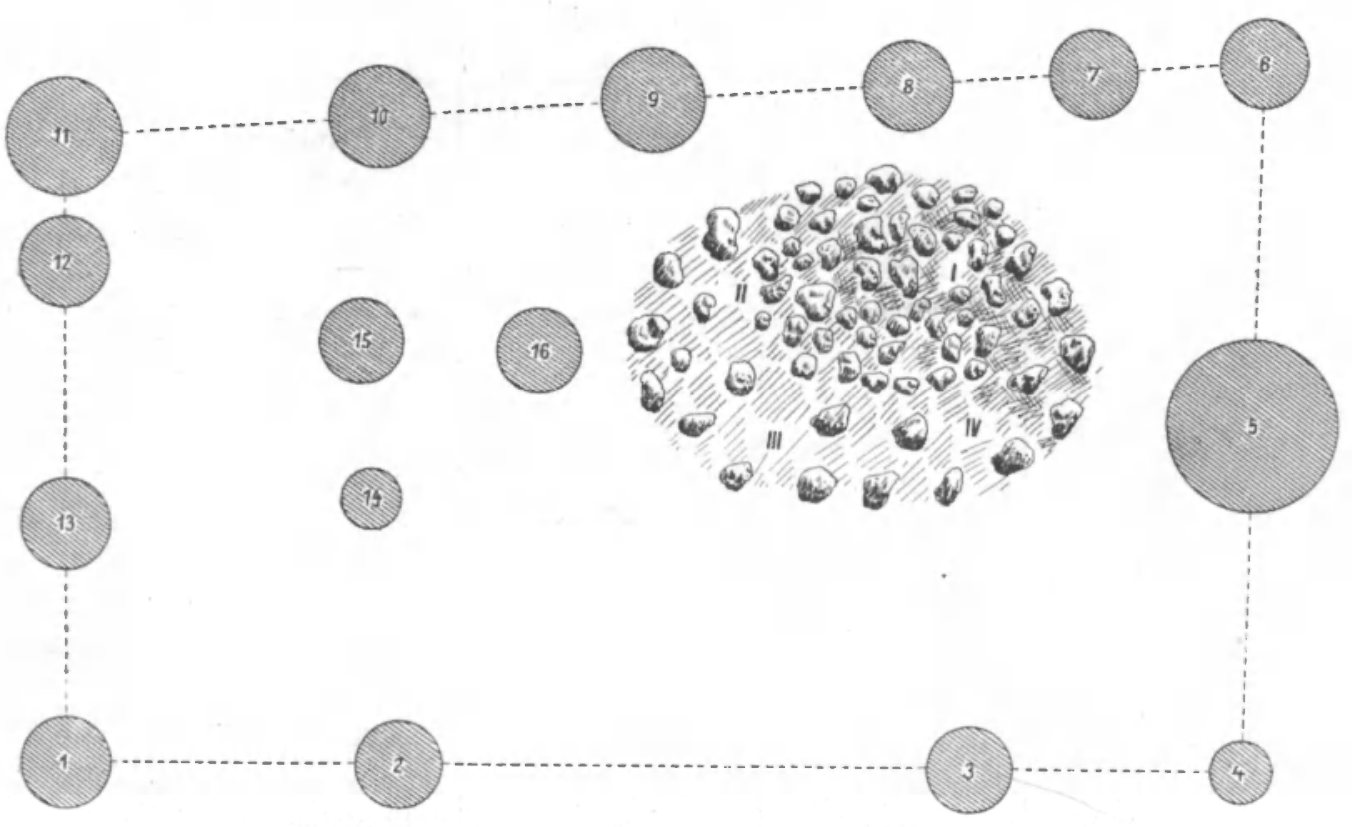

Abb. 2.

Der an der Nordostecke liegende Herd ist im Texte mit A bezeichnet, die Abfallgrube mit B.

andauernde Glut gelbrot gefärbt, eine Erscheinung, die man ja auch in Buch zu ungezählten Malen beobachten konnte.

Die $\mathrm{Pfos}$ tenlö cher hoben sich sämtlich im Horizontabschnitt deutlich vom Boden ab, allerdings nicht so scharf wie in Buch. Das hat einen zweifachen Grund. Der gewachsene Boden ist bei Hasenfelde nicht ganz so hell wie bei Buch, und der Inhalt der nach dem Einsetzen der Pfosten wieder zugeschütteten Pfostenlöcher kann hier nicht so tiefschwarz sein, weil er sich nur mit dem Humus vermischt hat, während man bei Buch 
zur Füllung der Pfostenlöcher den Brandschutt verwandte, der von früheren an derselben Stelle oder in der Nähe gestandenen Häusern herrührte. Die Pfostenlöcher bei Hasenfelde haben also mehr Ähnlichkeit mit den in Buch an der Peripherie der Ansiedlung liegenden, wo die Besiedlung schwächer war. Der Vertika ls chnit ter Pfostenlöcher unterscheidet sich von dem bei Buch gar nicht. Er zeigt ein unten oftmals etwas abgerundetes Rechteck. In einigen Fällen fanden sich auch hier in den Pfostenlöchern Steine vor, die zum Verkeilen des Pfostens gedient haben.

Ungefähr in der Mitte des Hauses stand ein Mittelpfosten, wie wir das von Buch her ja auch kennen.

Ich gebe im folgenden eine kurze Utbersicht, die zur Vergleichung mit dem Grundriss I bei Buch (Brandenburgia, Monatsblatt XVIII. 1910 S. 416 und Taf. I-III) dienen mag.

Grundriss I bei Hasenlelde, Kreis Lebus

\begin{tabular}{|c|c|c|c|}
\hline Pfosten & $\begin{array}{c}\text { Durchmesser } \\
\qquad \mathrm{cm}\end{array}$ & $\begin{array}{c}\text { Tiefe } \\
\text { (im gewachs. Boden) } \\
\text { rm }\end{array}$ & Inhalt \\
\hline 1 & 0,60 & 0,30 & i Steine \\
\hline$\underline{\underline{9}}$ & 1,50 & 0,30 & - \\
\hline 3 & $0, \overline{0} 0$ & 0,20 & ¿ Steine \\
\hline 4 & 0,40 & 0,35 & - \\
\hline$i$ & 1,20 & 0.40 & 9 Scherben \\
\hline 6 & 0.50 & 0,25 & - \\
\hline$\tau$ & $0,(0)$ & 0,25 & ; Steine \\
\hline 8 & 0,60 & 0,35 & 3 Scherben \\
\hline 9 & 0.70 & 0,40 & ¿ Scherben \\
\hline 10 & $0, \pi(1)$ & 0,411 & - \\
\hline 11 & $0, \pi 0$ & 0,35 & 1 Scherben \\
\hline 12 & 0.60 & 0.55 & - \\
\hline 13 & 0,60 & 0,30 & 1 Scherben \\
\hline 14 & 0,40 & 0,40 & - \\
\hline 15 & $0, \overline{0} 0$ & 0.40 & 3 Scherben \\
\hline 16 & 0,60 & $0,3 j$ & - \\
\hline $1 i$ & 0,75 & 0,35 & 12 Steine \\
\hline is & 1,20 & 0,30 & $\begin{array}{c}1 \text { Scherben, } \\
\text { Holzkohle }\end{array}$ \\
\hline
\end{tabular}

In unmittelbarster Nähe des Hauses lag e in z we it e r Herd (A), in dessen Umgebung keine Pfostenlöcher zu finden waren, die unbedingt zu ihm gehören müssten. Dieser Herd war elliptisch, 2,40 $m$ lang, $1,65 \mathrm{~m}$ breit, sorgfältig aus Steinen gepackt (es wurden noch 381 gezählt) und enthielt ebenfalls viel Holzkohle, zahlreiche Gefässreste (57) und Tierknochen (13).

Fast genau in der Mitte des Herdes stand noch der untere Teil eines rohen Kochtopfes in ursprünglicher Lage (Abb. 5a). 


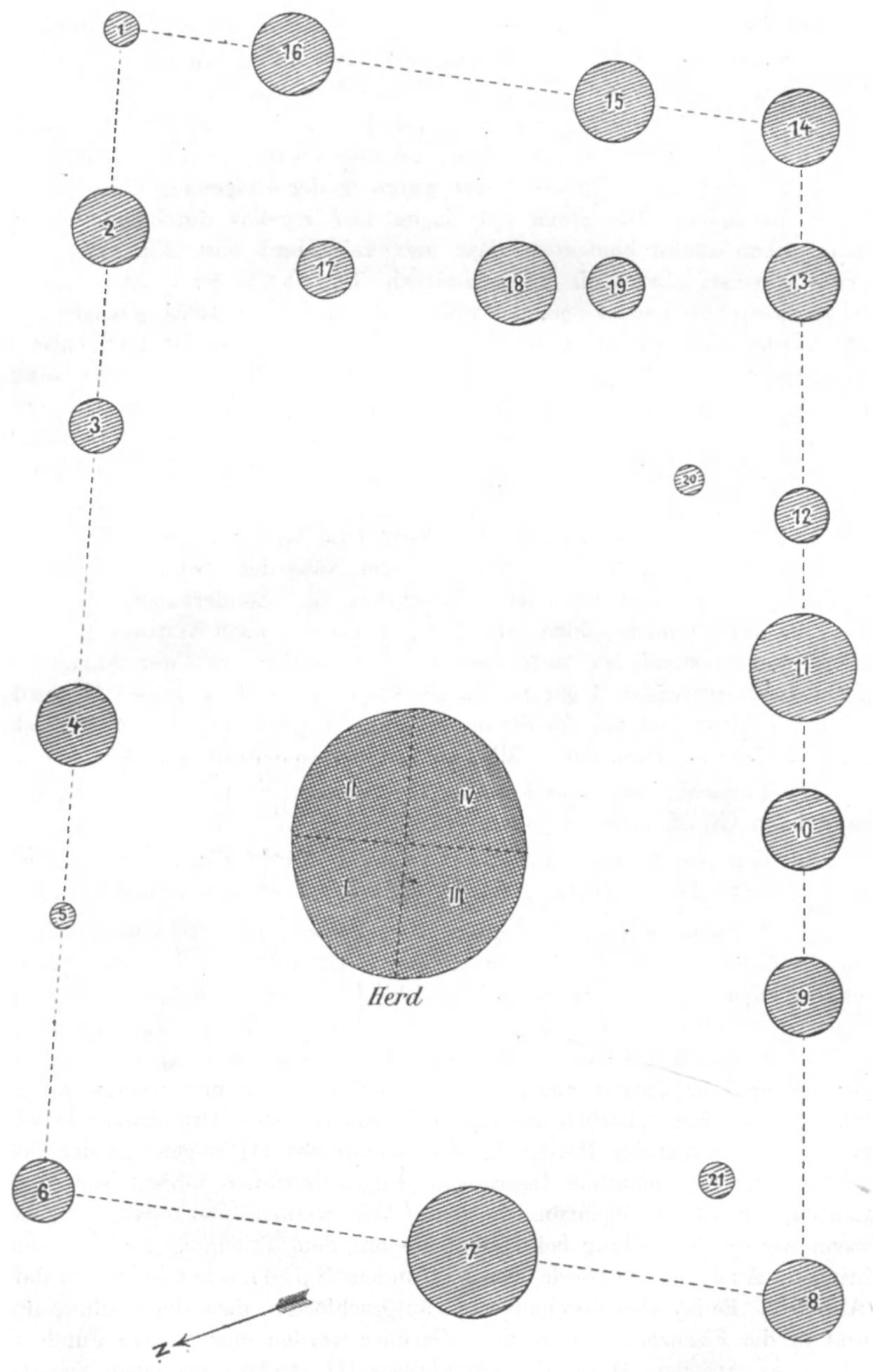

Hasenfelde, Grundriß II.

Abb. 3. 
Ausserdem wurden auf dem Herde $\mathrm{z}$ we i E i e r- oder Sch wa l bens teine, ein Stückchen Wandbewurf aus Lehm, mit Stroh gemischt, und ein doppelkonischer $\mathrm{Sp}$ in $\mathrm{n}$ wirtel gefunden.

In einiger Entfernung von Grundriss I (etwa $35 \mathrm{~m}$ nach Norden) wurde eine $\mathrm{A}$ b f a llg rube (B) aufgedeckt, die aber ebenso gut zu einem anderen Hause gehören kann. Pfostenlöcher waren in der Umgebung dieser Grube nicht vorhanden. Die Steine (34) lagen hier regellos durcheinander, was allein schon darauf hindeutet, dass hier kein Herd war. Für eine Abfallgrube sprechen aber auch die zahlreichen Tierknochen (90) und der $S t$ irnzapfen einer Ziege, der wohl kaum auf einen Herd gelangen wird. Die Grube war verhältnismässig flach, $30 \mathrm{~cm}$ unter dem Planum, also im ganzen etwa $70-80 \mathrm{~cm}$ tief. In der Grube fanden sich noch ein bearbeiteter und Benutzungsspuren aufweisender Stein, ein grösserer Feuersteinsplitter e in Eierstein, zwei ebenfalls mit Stroh gemischte Lehmbewurf -, $\mathrm{st}$ ü $\mathrm{cke}$ und 32 Tongefässreste, darunter sechs Rand- und vier Bodenstücke.

Die Grundris se II und III (Abb. 3. 4) lagen nur etwa 20 Schritte roneinander entfernt am „Oberpark", in der Nähe der "Schafwäsche“, eines Pfuhles, der auch heute noch Wasser enthält. Sonderbarerweise ist die Vorhalle von II nach Südost, die von III dagegen nach Nordwest gerichtet. Herd- und Pfostenlöcher unterschieden sich in der Art ihrer Anlage von denen des Grundrisses I gar nicht. Beim Herd des Grundrisses II wurden 118, bei dem von III 95 Steine gezählt. Die Füllung der Pfostenlöcher war auch hier in den meisten Fällen gelbgrau, seltener grau oder gar schwarz.

Die Pfostenlöcher 20 und 21 bei II erinnern an die Stützen der Ruhebank beim Grundriss 87 in Buch. (P. Z. II, $396 \mathrm{f}$. Abb. 11.)

Auf dem Herde des Grundrisses III wurde ein Feuersteinsplitter gefunden, der das Bruchstück eines prismatischen Messers sein dürfte.

Die Wohnstätten am Radelandsberge gehören der jüngeren Bronzezeit an. Die Gef äss r e ste, die in den übrigen Furdstellen lagen, waren fast aus schliesslich unverziert. Es ist daher nicht leicht, die Grundrisse einer engbegrenzten Zeit zuzuweisen. Die unverzierte Tonware stimmt jedoch zum grossen Teile überein mit der unverzierten Ware aus dem der jüngeren Bronzezeit angehörenden Friedhofe, der nur wenige Minuten entfernt ist. Ein Scherben aus einem Pfostenloch des Grundrisses I, sowie zwei Scherben aus der Herdgrube des Grundrisses III zeigen auf der Oberfläche jene mit scharfem Instrument eingeschnittenen Linien, wie sie der Lausitzer Keramik eigentümlich sind. Wir werden also nicht fehlgehen wenn wir die Ansiedlung bei Hasenfelde mit dem Friedhofe in Verbindung bringen. Auch der im Herde von I gefundene Spinnwirtel spricht dafür (Abb. 5c). Es ist aber durchaus nicht ausgeschlossen, dass die Siedlung noch weit in die Eisenzeit hineinreicht. Darüber werden uns weitere Funde belehren. - Auf dem Herde des Grundrisses III fanden wir einen Scherben mit eigenartigen Zeichen (Abb.5b). Man möchte an die wendische Vellenlinie denken, so nachlässig sind die Zeichen in den Ton gekritzelt. 


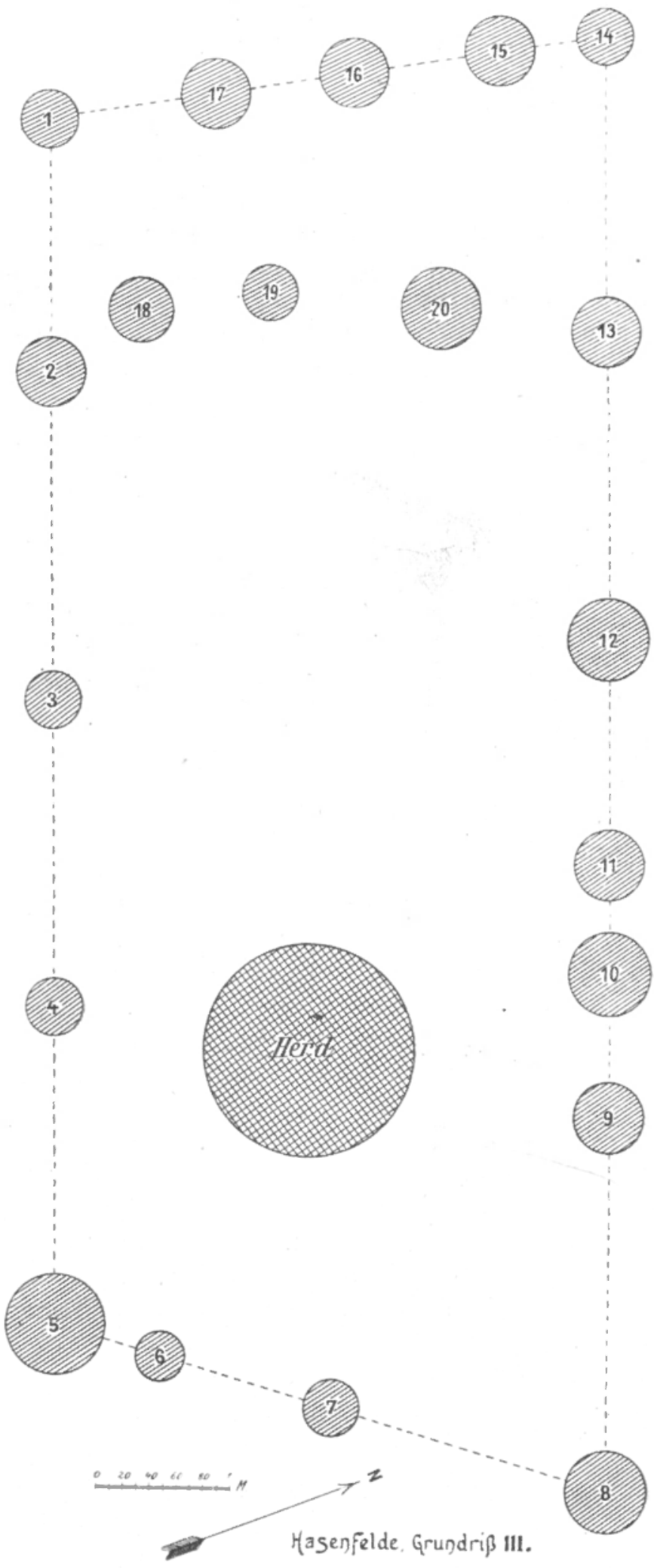

Abb. 4. 
$\mathrm{Ob}$ es sich bei Hasenfelde auch um Spuren einer wendischen Siedlung handelt, wage ich noch nicht mit voller Sicherheit zu entscheiden. Sollte es der Fall sein, so würde das Problem dadurch nur noch interessanter.

Als Ử berreste der Wände sind nur drei Leh m bew urf t ü cke übrig geblieben, die sich von den bei Buch gefundenen wesentlich unterscheiden. Keines dieser Stücke zeigt den Abdruck eines Rundholzes. $\mathrm{Ob}$ das Zufall ist oder auf abweichende Bauart der Wände deutet, kann nur die weitere Untersuchung lehren. Aber auch in anderer Beziehung unterscheiden sich die Bewurfstücke von denen in Buch. Dort ist der Lehm stets mit Steinchen gemischt, nur ein einziges Stückchen - unter Hunderten - enthielt etwas Stroh. Hier bei Hasenfelde sind alle [drei Stücke mit ganz klein $\mathrm{zerhack}$ e m Stroh vermischt und zwar ist es wie der deutliche Addruck eines Kornes beweist - in dem einen Falle Weizenstroh.

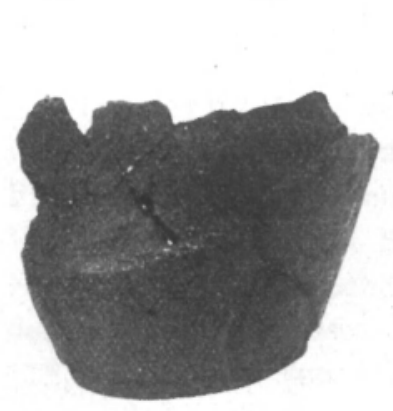

a

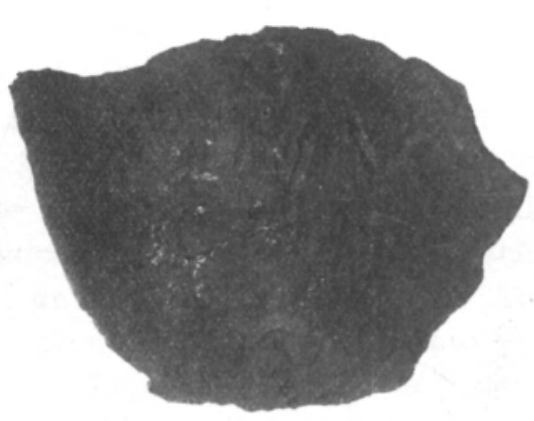

b

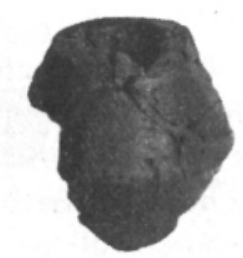

c

Abb. 5. a-c. Gefässbruchstücke und Spinnwirtelfragment von Hasenfelde, Kr, Lebus. Etwa $1 / 2$ nat. Gr. - Märkisches Museum, Berlin.

Die Untersuchung der Bewurfstücke und der Gefässreste mit dem Mikroskop hat sich äusserst ergiebig gezeigt. Es liessen sich eine grosse Menge von $\mathrm{Abdr}$ ü $\mathrm{k}$ en feststellen, von den immerhin groben Eindrücken der Getreidekörner, unter denen namentlich auch $\mathrm{Hirse}$ häufig vertreten ist, bis zu den feinsten Blütenspelzen der Gräser. Diese Untersuchungen sind noch nicht abgeschlossen. Die Ergebnisse werden später veröffentlicht werden.

Die Bedeutung der Hasenfelder Hausfunde liegt darin, dass die Beobachtungen bei Buch bestätigt werden. Zugleich aber werden uns neue Probleme gestellt bezüglich der Bauart der Häuser. Dass diese Probleme nur mit dem Spaten zu lösen sind, hat man ja durch die Erfahrungen der letzten Zeit gelernt.

Die Hasenfelder Funde sind in diesem Sinne nicht nur eine Bestätigung der Bucher, sondern zugleich ihre wichtige Ergänzung. 